\title{
Macro Matters: Der Makroökonomische Dialog der Europäischen Union
}

Die makroökonomische Politik steht wieder im Zentrum der wirtschaftswissenschaftlichen und wirtschaftspolitischen Debatte. Ihre „Renaissance“, die sich in der Wissenschaft bereits seit einigen Jahren abzeichnete, tritt in der weltweiten Finanzmarkt- und Wirtschaftskrise auch in der Wirtschaftspolitik offen zutage. Geldpolitik und Finanzpolitik tragen die Hauptlast bei der Stabilisierung des Finanzsystems und der Kompensation des weltweiten Nachfrageeinbruchs. Zur Krisenbewältigung gerät die Makropolitik wieder in die Rolle aktiv gestaltender Politik. Aber bedurfte es erst einer Krise, um (wieder) zu der Erkenntnis zu kommen: macro matters?

\section{1 \\ Einleitung}

Es war in wesentlich ruhigerem weltwirtschaftlichen Fahrwasser, als Anfang Juni 1999 der Makroökonomische Dialog (MED) der Europäischen Union (EU) unter deutscher Präsidentschaft vom Europäischen Rat beschlossen wurde. Er wird demnach zehn Jahre alt; dennoch ist er immer noch ein wenig bekanntes Element der Architektur der wirtschaftspolitischen Koordinierung in der EU. Das kann weder an seinem wirtschaftspolitischen Potenzial noch an seiner Besetzung liegen. Die Besetzung ist hochkarätig und der mögliche Beitrag zu den zentralen gesamtwirtschaftlichen Zielen Wachstum, Beschäftigung und Preisstabi- lität kaum zu überschätzen. Es liegt wohl eher an der selbst verordneten Vertraulichkeit des Dialogs, dass er nicht den Bekanntheitsgrad hat, den er zumindest von seinem Anspruch her verdient. Manche Kritiker legen ihm das als Schwäche aus, andere sehen gerade darin seine Stärke. ${ }^{2}$ Der Beitrag zieht eine Zwischenbilanz des Makroökonomischen Dialogs. Zunächst rekapituliert er die Überlegungen, die zur Einrichtung und Ausgestaltung des MED geführt haben (Abschnitt 2-4). Anschließend werden Sichtweise und Interessenlage der beteiligten Akteure gegenübergestellt (Abschnitt 5). Ausgehend von einer Bewertung der bisherigen Wirkungen (Abschnitt 6) werden Vorschläge zur institutionellen Stärkung und inhaltlichen Verbesserung des MED präsentiert (Abschnitt 7).

\section{Abb. 1: Beschäftigung im Euroraum-12, 1961 bis 2008}

- Veränderungen in \% -



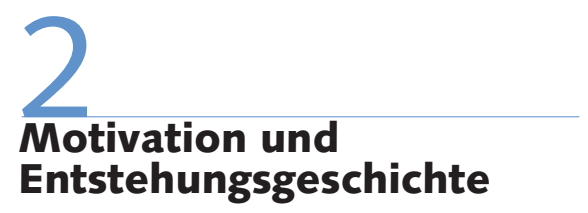

Ein Blick zurück zeigt, dass die Beschäftigungsdynamik im Euroraum insbesondere 1967, 1974/75, 1981/83, 1991/93 und 2001/03 schockartig eingebrochen ist.

Diese abrupten Beschäftigungseinbrüche waren jeweils das Ergebnis exogener Schocks und der anschließenden Reaktion der makroökonomischen Politiken auf diese Schocks. Ganz überwiegend war es ein Konflikt zwischen Lohnentwicklung und

1 Die Autoren geben hier ihre persönliche Meinung wieder. W. Koll war seit Beginn im Herbst 1999 bis Herbst 2006 Vorsitzender des Makroökonomischen Dialogs (MED) auf technischer Ebene und Berichterstatter im MED auf politischer Ebene. V. Hallwirth war jeweils an den inhaltlichen Vorbereitungen beteiligt.

2 Zur Entstehung und Zwischenbilanz des MED vgl. Koll (2005) sowie die anderen dort enthaltenen Beiträge, insbes. von Janssen und Watt, sowie kürzlich Hallwirth/Koll (2009) und die dort angegebene Literatur.

Willi Koll, Dr., ist Ministerialdirigent im Bundesministerium für Wirtschaft und Technologie. Arbeitsschwerpunkte: Gesamtwirtschaftliche Entwicklung, Analysen und Projektionen.

e-mail:willi.koll@bmwi.bund.de Volker Hallwirth, Dr., ist Regierungsdirektor im Bundesministerium für Wirtschaft und Technologie. Arbeitsschwerpunkte: Gesamtwirtschaftliches Wachstum und Demografie. e-mail: volker.hallwirth@bmwi.bund.de 
Geldpolitik, der die Konjunktur von der Nachfrage- und Angebotsseite in die Zange nahm. Die Gleichzeitigkeit von nicht stabilitätsgerechter Lohnentwicklung und starker monetärer Restriktion stoppte abrupt die wirtschaftliche Dynamik und verursachte teilweise massive Einbrüche von Produktion und Beschäftigung.

Die wirtschaftspolitische Schlussfolgerung aus diesen Entwicklungsmustern lag auf der Hand: Konflikte von Lohnentwicklung und Geldpolitik sollten künftig vermieden werden. Das Ziel war, das oftmalige Gegeneinander der wirtschaftspolitischen Akteure durch ein Miteinander zu ersetzen. Kooperation statt Konfrontation sollte Verlierer zu Gewinnern machen.

Ende der 1990er Jahre gab es ein Zeitfenster für die institutionelle Umsetzung dieser Konzeption. Die letzte Stufe der Wirtschafts- und Währungsunion (WWU) sollte Anfang 1999 in Kraft treten. Der Übergang zur gemeinsamen Geld- und Währungspolitik bei weiterhin dezentralen Verantwortlichkeiten für die Finanzpolitik, die Strukturpolitiken sowie die Lohnentwicklung erforderten eine neue Architektur der wirtschaftspolitischen Koordinierung in der EU und im Euroraum, die in den Verträgen von Maastricht und Amsterdam verankert wurde. Bereits 1997 wurde die Einfassung der nationalen Finanzpolitik in den europäischen Stabilitäts- und Wachstumspakt beschlossen. Um die anspruchsvollen Ziele der Europäischen Union - u. a. ein „hohes Beschäftigungsniveau“ und „beständiges, nichtinflationäres Wachstum" (Art. 2, EGVertrag) - zu erreichen, vereinbarten die Mitgliedstaaten, ihre Wirtschaftspolitik als eine „Angelegenheit von gemeinsamem Interesse" zu betrachten und im Rat anhand der sogenannten „Grundzüge der Wirtschaftspolitik der Mitgliedstaaten und der Gemeinschaft" zu koordinieren (Art. 99, EG-Vertrag). Mit dem Vertrag von Amsterdam 1997 wurde dem EG-Vertrag ein eigenes Kapitel „Beschäftigung“ hinzugefügt. Eine „Koordinierte Beschäftigungsstrategie“ wurde durch jährliche „Beschäftigungspolitische Leitlinien“, „Nationale beschäftigungspolitische Aktionsprogramme" sowie deren Prüfung durch einen "Gemeinsamen Beschäftigungsbericht" im Rahmen des sogenannten Luxemburg-Prozesses verwirklicht. Über den Arbeitsmarkt hinaus wurde ein Jahr später auf dem Europäischen Rat (ER) von Cardiff die Koordinierung auf Reformen in allen strukturpolitischen Bereichen ausgedehnt.
Die strukturpolitische Aufgabe war demnach durch die Luxemburg- und Cardiff-Agenda weitgehend abgedeckt; eine Erweiterung der Agenda um eine sozialpolitische und umweltpolitische Komponente kam in späteren Jahren hinzu.

Es fehlte die makroökonomische Dimension. Zu ihrer Ausfüllung hatte der ER Wien im Dezember 1998 den Auftrag erteilt. Die Umsetzung lag in der Hand der deutschen Präsidentschaft im ersten Halbjahr 1999; sie wurde durch den Europäischen Rat Anfang Juni 1999 in Köln in Gestalt eines "Makroökonomischen Dialogs“ beschlossen. Der ER Köln hatte dabei zugleich die drei Politikbereiche Koordinierte Beschäftigungsstrategie, Strukturreformen und Makroökonomischer Dialog zu einem „Europäischen Beschäftigungspakt" zusammengefügt. Hierdurch sollten die Wechselwirkungen der drei Elemente sichtbar und nutzbar gemacht werden: jedes für sich war notwendig, aber nicht hinreichend, alle drei zusammen verstärkten die Wirkung eines jeden. Nur ein Jahr später wurde der Europäische Beschäftigungspakt in die Form der sogenannten Lissabon-Strategie gebracht. Strukturreformen, sozialer Zusammenhalt und ein „geeigneter makroökonomischer Policy-mix" sollten die EU sowohl ,zum wettbewerbsfähigsten und dynamischsten wissensbasierten Wirtschaftsraum der Welt" machen als auch sie in die Lage versetzen, „wieder die Voraussetzungen für Vollbeschäftigung zu schaffen". Die EU setzte sich darüber hinaus ehrgeizige Beschäftigungsziele bis zum Jahr 2010. ${ }^{3}$

\section{Institutionalisierung}

Wie oben skizziert, war es zu massiven Konflikten zwischen den Akteuren der makroökonomischen Politik selbst unter Bedingungen gekommen, in denen die Kompetenz für die makroökonomischen Instrumente noch voll in der Hand der $\mathrm{Na}$ tionalstaaten lag. Aufgrund der Übertragung von Kompetenzen im Bereich der makroökonomischen Politiken auf die europäische Ebene hatten sich die institutionellen Voraussetzungen zur Vermeidung bzw. Entschärfung derartiger Konflikte erheblich kompliziert. Mit der gestiegenen Zahl der makroökonomischen Akteure der Euroraum umfasste damals elf Mitgliedstaaten - erhöhte sich auch die Zahl der Freiheitsgrade für derartige Konflikte. Auch wenn die gestiegene Komplexität des Zusammenwirkens der Geldpolitik mit den nationalen Finanzpolitiken und Lohnentwicklungen zunächst nur innerhalb der WWU von Bedeutung war, sollte bereits eine Lösung vorgezeichnet werden, die auch die Mitgliedstaaten der EU einschloss, die noch über die volle makroökonomische Autonomie verfügten. In Ausfüllung des makroökonomischen Pfeilers war es deshalb Ziel des MED, alle makroökonomischen Akteure in der EU an einen Tisch zu bringen, um über den Dialog zu einer gemeinsamen Analyse und zu einem konsistenten Policy-Mix zu gelangen. Zwar gab es mit dem ECOFIN-Rat (Rat für Wirtschaft und Finanzen) bereits ein Gremium zur Abstimmung innerhalb der Finanz- und Wirtschaftspolitik. Mit der Einrichtung der informellen Eurogruppe zu Beginn der WWU wurde auch die Geldpolitik stärker einbezogen. Aber erst mit dem MED fand auch die lohnpolitische Dimension Eingang in eine „weiche" Form der Koordinierung.

Dies blieb nicht unstrittig. Kritiker wandten ein, dass eine „Lohnpolitik“ auf EU- oder WWU-Ebene angesichts der vielfältigen Lohnfindungsformen in jedem Mitgliedstaat und der Autonomie der nationalen Tarifparteien unmöglich sei. Dies sprach jedoch nicht gegen die Einbeziehung der Sozialpartner. Fraglos erhöhte dieser Ansatz aber die Anforderungen an ihre interne Koordinierung, um sich nach außen im MED als makroökonomische Akteure präsentieren zu können. Die Teilnahme der Sozialpartner ist aber nicht nur zur Vermeidung der zuvor genannten Konflikte unabdingbar. $\mathrm{Zu}$ den wichtigsten Spielregeln der WWU gehört, dass in allen Mitgliedstaaten eine stabilitätsgerechte Lohnentwicklung verfolgt wird, um ein Überschießen in inflatorische oder deflatorische Richtung zu vermeiden. Auch dieser Punkt zählt zur Agenda des MED.

Gegen Koordination generell wurde eingewandt, dass jeder makroökonomische Akteur selber wisse, was er zu tun habe, es einer Koordination daher nicht bedürfe und eine solche nur zur Verwischung von Verantwortlichkeiten beitrüge. Dem ist entgegenzuhalten, dass tendenziell das

\footnotetext{
3 Zur umfassenden Behandlung der Thematik des Abbaus der Arbeitslosigkeit und der Wiedergewinnung der Vollbeschäftigung vgl. u. a. Hallwirth (1998); Horn (2005); Holtfrerich (2007); Flassbeck/Spiecker (2007)
} 
Handeln jedes makroökonomischen Akteurs weder in Bezug auf die Ziele der jeweils anderen neutral noch in Bezug auf sein eigenes Ziel autark ist - es sei denn, man nähme wie z.B. im Falle der Geldpolitik hohe Stabilisierungskosten in Kauf. Diese externen Effekte sowie die bisherigen Erfahrungen mit Konflikten zwischen Lohn- und Geldpolitik lassen nicht erwarten, dass eine klare Zuweisung von Verantwortlichkeiten ausreicht, um ein Paretooptimales Ergebnis zu erreichen. Die Chancen für ein solches Ergebnis sind deutlich höher zu veranschlagen, wenn im Rahmen eines Dialogs, wie ihn der MED vorsieht, der ernsthafte Versuch unternommen wird, über klare Signale zwischen den Akteuren Missverständnisse zu vermeiden, Erwartungen zu stabilisieren und wechselseitiges Verständnis und Vertrauen aufzubauen. Bei Herausbildung einer möglichst gemeinsamen Sicht, wie Lage und Perspektiven zu beurteilen sind, kann auch Klarheit darüber gewonnen werden, was wirtschaftspolitisch zu tun oder zu lassen ist.

Bei bereits damals 15 Mitgliedstaaten und - neben der EZB - noch vier weiteren Zentralbanken musste bei der Zusammensetzung des MED eine Auswahl getroffen werden, sollte ein Dialog möglich sein. So wird die Fiskalpolitik durch eine Troika in Gestalt des amtierenden Präsidenten des ECOFIN-Rates, der zugleich auch Vorsitzender des MED ist, und seine zwei Nachfolger vertreten. Die Arbeitsminister sind über ihren jeweiligen Ratsvorsitzenden und seinen Nachfolger beteiligt. Entsprechend nehmen von der Kommission die beiden Kommissare für Wirtschaft und Währung sowie für Beschäftigung teil. Die Geldpolitik sitzt in Gestalt des Präsidenten der EZB und eines Vertreters der Nicht-Eurozentralbanken am Tisch. Die Sozialpartner nehmen mit jeweils vier Vertretern der Spitzenorganisationen der Arbeitgeber und der Gewerkschaften teil. Seit einigen Jahren ist auch der Vorsitzende der Eurogruppe ständiges Mitglied des MED. Hinzu kommen die Vorsitzenden des Wirtschafts- und Finanzausschusses (WFA), des Wirtschaftspolitischen Ausschusses (WPA) und des Beschäftigungspolitischen Ausschusses sowie des MED auf technischer Ebene. Damit setzt sich der MED aus rund 20 Personen zusammen, die zweimal jährlich zusammentreffen. Jeder MED auf politischer Ebene wird in entsprechender Zusammensetzung durch eine ganztägige Sitzung auf technischer Ebene vorbereitet, über deren Ergebnisse dem MED einleitend berichtet wird.

Wie vom ER Köln festgelegt, ist die Tagesordnung des MED für jede Sitzung von aktuellen Nuancierungen abgesehen die Gleiche: Gedankenaustausch über die wirtschaftliche Lage und Perspektiven und über die daraus folgenden Herausforderungen für die Makropolitik. Jede Seite trägt dabei einleitend vor, was sie selbst zur Bewältigung der Probleme beiträgt und was sie dazu von den jeweils anderen Akteuren erwartet. Die Beratungen sind vertraulich; es gibt keine bindenden Beschlüsse und kein Protokoll.

Dabei besteht die Erwartung, dass jeder der Beteiligten über Verlauf und Ergebnisse an die von ihm vertretene Institution zurückberichtet, so z.B. die anwesenden Finanzminister an den ECOFIN-Rat bzw. die Eurogruppe oder die Vertreter der Arbeitgeber und Gewerkschaften an ihre Organisationen.

\section{4 \\ Wissenschaftliches und politisches Umfeld}

Der MED hatte seit seinem Beginn den EUkontinentalen wissenschaftlichen „mainstream" und den dadurch beeinflussten wirtschaftspolitischen Konsens gegen sich. Bis auf das kurze Zeitfenster gesteigerter makroökonomischer Sensibilität, das zur Etablierung des MED geführt hatte, fehlte jeder paradigmatische Rückenwind. Die überwiegende Sicht und Ausrichtung der Makroökonomie waren mehr auf Enthaltung und Vermeidung als auf aktive Gestaltung gerichtet. Die Gestaltungskraft konzentrierte sich fast ausschließlich auf die Strukturreformagenda. Die Rolle der Makroökonomie wurde im Zuge der Neuausrichtung der Lissabon-Strategie 2005 auch formal zugunsten der Strukturagenda weiter zurückgenommen. Die makroökonomische Dimension selbst wurde noch stärker auf die Wahrung von Preisstabilität und die Einhaltung des (reformierten) Stabilitäts- und Wachstumspaktes verkürzt. Dabei ist unbestritten, dass die Erhaltung von Preisstabilität eine unabdingbare Voraussetzung für einen lang anhaltenden, dynamischen Aufschwung ist. Auch ist die Wahrung der langfristigen Tragfähigkeit der öffentlichen Finanzen ein hohes wirtschaftspolitisches Gut. Ebenso ist unbe- stritten, dass strukturelle Reformen in vielen Bereichen zu positiven Wachstumseffekten beitragen. Unbestritten ist auch, dass die Reformagenda eine Daueraufgabe bleibt, um verbliebene Steuerungsfehler, fehlende oder falsche Anreize, Sanktionen, Transparenz und Kontrolle durch „better regulation" zu ersetzen. Nichts belegt diesen Befund mehr als die Tatsache, dass sich eine Finanzmarktkrise im derzeitigen Ausmaß überhaupt hat entwickeln können.

Strukturreformen können makroökonomische Gestaltung aber niemals ersetzen. Diese theoretisch und empirisch gut belegte Einsicht hat sich in der derzeitigen Krise rasch zur Konsensmeinung entwickelt. Erfolgreiche makroökonomische Politik und erfolgreiche Strukturreformen schließen sich dabei nicht aus. Eine beschäftigungs- und stabilitätsorientierte Makropolitik und Strukturreformen zur Stärkung von Produktivität, Innovation und Qualifikation können und sollten sich vielmehr wechselseitig bedingen, ergänzen und verstärken.

\section{Makroökonomische Akteure}

Neben dem allgemeinen wissenschaftlichen und politischen Umfeld sind Sichtweise und Interessenlage der einzelnen makroökonomischen Akteure für die Positionierung im MED und für das realwirtschaftliche Ergebnis entscheidend:

- Für die Geldpolitik ist vor allem ihr Mandat nach Art. 105 Absatz 1 EG-Vertrag maßgeblich. „Das vorrangige Ziel des ESZB ist es, die Preisstabilität zu gewährleisten. Soweit dies ohne Beeinträchtigung des Zieles der Preisstabilität möglich ist, unterstützt die Geldpolitik die allgemeine Wirtschaftspolitik in der Gemeinschaft, um zur Verwirklichung der in Artikel 2 festgelegten Ziele der Gemeinschaft beizutragen." Das Mandat enthält also eine klare Abfolge der Ziele. Dabei wird der Satz 2 des Art. 105 Absatz 1 durch die geldpolitischen Akteure überwiegend so interpretiert, dass seiner Zielsetzung am besten und ausschließlich durch die Umsetzung von Satz 1 gedient ist. Überdies ist vorherrschende Meinung, dass Inflation und damit die Verletzung des Preisstabilitätsziels nur bei einer übermäßigen monetären Expansion möglich sind. 
Mandat und mehrheitliche Sichtweise sprechen deshalb beide für eine tendenziell weniger expansiv ausgerichtete Geldpolitik. Das gilt insbesondere dann, wenn von den geldpolitischen Instanzen die Verletzung der Preisstabilität nach oben höher gewichtet werden sollte als eine Verfehlung nach unten.

- Die Gewerkschaften werden stark von der Einkommensverteilung geleitet. Soweit es um die Wahrung der Verteilungsposition geht, sollte der Anstieg der effektiven Nominallöhne dem Anstieg der Produktivität und des Preisniveaus entsprechen. Je besser die Lage auf dem Arbeitsmarkt, desto stärker ist die Verhandlungsposition der Gewerkschaften. Auch deshalb sind die Gewerkschaften zugleich an einem möglichst hohen Beschäftigungsstand interessiert, wie ihn Art. 2 EG-Vertrag zum Ziel erhoben hat.

Darüber, wie mehr Beschäftigung erreicht werden kann, gibt es in der Wissenschaft ein Spektrum unterschiedlicher Sichtweisen. Das eine Extrem betont den Kostenaspekt der Löhne und erwartet deshalb mehr Beschäftigung davon, dass die Lohnentwicklung deutlich unterhalb des Verteilungsspielraums gehalten wird. Das andere Extrem betont die Rolle der Kaufkraft der Löhne und verbindet diese Sicht mit möglichst hohen Lohnabschlüssen. Beide Positionen fokussieren jedoch nur auf Teilaspekte und greifen deshalb zu kurz. Zumindest muss die mögliche Reaktion der Geldpolitik auf inflatorische Abschlüsse mit einbezogen werden. Das bedeutet, wenn nicht die Lohnforderung, so doch das Ergebnis auf Abschlüsse zu begrenzen, die im Einklang mit dem mittelfristigen Produktivitätswachstum und dem Preisziel der EZB stehen. Dabei ist zu sehen, dass stabilitätskonforme Lohnstückkosten zwar zur Wahrung des Preisziels, aber ohne eine z.B. makropolitisch angestoßene, Stärkung der Nachfrage nicht zugleich auch zu mehr Beschäftigung beitragen. Bereits Keynes hatte jedoch betont, dass ein „monetary management by the trade unions", also eine Erhöhung der realen Geldmenge durch sinkende Lohnstückkosten und damit sinkende Preise, keine empfehlenswerte Strategie zur Erhöhung der realen Nachfrage darstellt. Auch gesamtwirtschaftlich wirksame Anreize für eine Verbesserung der für die Lohnhöhe entscheidenden Produktivitätskomponente, z.B. durch eine investitionsgetriebene Verbesserung der Kapitalausstattung („capital deepening"), liegen bei Weitem nicht allein in der Hand der Tarifparteien. Im Ergebnis sind die Gewerkschaften zur Erreichung ihrer Ziele stark auf die Unterstützung durch die anderen Akteure angewiesen.

- Die Position der Arbeitgebervertreter sollte eigentlich ähnlich gelagert, d.h. auf die Stärkung der gesamtwirtschaftlichen Dynamik gerichtet sein, denn ebenso wie diese für hohe Löhne und mehr Beschäftigung sorgt, ermöglicht sie zugleich hohe Gewinne und mehr Investitionen. In der Praxis scheint allerdings die Position der Arbeitgebervertreter überwiegend von der Sicht der von ihnen vertretenen Unternehmen, also einzelwirtschaftlich geleitet zu sein. - Die Fiskalpolitik hat primär eine allokative und distributive Funktion. Für die konjunkturelle Entwicklung hat sie - abgesehen von Ausnahmezuständen wie derzeit - eher eine passive Funktion, indem sie die automatischen Stabilisatoren wirken lässt. Zudem ist sie an die Einhaltung des Europäischen Wachstums- und Stabilitätspakts gebunden.

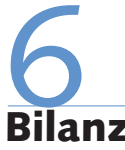

Was hat der MED gebracht? Eine Bewertung muss sich insbesondere an der tatsächlichen wirtschaftlichen Entwicklung seit Beginn des MED orientieren. Diese lässt sich kurz wie folgt zusammenfassen:

- Anders als in den Jahrzehnten zuvor hat es keine überschießende Inflation gegeben. Auch konnte eine tiefe Rezession nach dem Ölpreisanstieg und dem Platzen der „New Economy-Blase“ 1999/2000 vermieden werden.

- Die Beschäftigungsdynamik ist im Euroraum ab 2001 insgesamt aber in ähnlichem Maß eingebrochen wie z. B. 1981/83. Die Arbeitslosigkeit blieb vergleichsweise hoch. Auch die angestrebte deutliche Rückführung der Staatschulden in Relation zum Bruttoinlandsprodukt (BIP) gelang nicht.

Im Ergebnis wurde das angebotsseitige Potenzial des Euroraums nicht ausgeschöpft. Das Potenzialwachstum, die zentrale Zielgröße der sogenannten „Lissabon-Strategie", nahm nicht wie erhofft zu, sondern ging im Trend schon vor dem Ausbruch der weltweiten Finanzmarkt- und Wirtschaftskrise zurück. Darüber hinaus entwickelten sich innerhalb des Euroraums permanente und persistente Divergenzen, gemessen an den Veränderungsraten bei Nominallöhnen, Lohnstückkosten, Verbraucherpreisen, realem Wachstum, Beschäftigung und Leistungsbilanzsalden. So hatte ein Land wie z.B. Spanien im Vorfeld des Beitritts seinen Notenbankzins auf das Niveau der WWU herabgeschleust. Allein aufgrund dieses starken monetären Impulses lagen zu Beginn der WWU die Wachstums- und Beschäftigungsdynamik, aber auch die Inflationsrate des Landes deutlich oberhalb des WWU-Durchschnitts. Hierdurch wurde ein Kreislauf angetrieben und in Bewegung gehalten, der eine durch Marktprozesse getragene Anpassung an eine stabilitätsorientierte Entwicklung über lange Zeit verhinderte. So ermöglichten starkes reales Wachstum und eine hohe Beschäftigung die Durchsetzung relativ hoher Lohnanstiege, die die Nachfrage, aber im Zuge von Kostenüberwälzungen auch die Inflation weiter antrieben. Insbesondere ein Anstieg der Immobilienpreise stimulierte seinerseits fortgesetzt Nachfrage und Wachstum. Hohe reale Zuwachs- und Inflationsraten verschafften den öffentlichen Haushalten stark steigende Einnahmen, die in entsprechend steigende, prozyklisch wirkende Staatsausgaben fließen konnten, ohne in Konflikt mit dem Maastricht-Kriterium zu geraten. Die Geldpolitik konnte dieser Entwicklung keinen Einhalt bieten. Denn eine nationale Geldpolitik gab es nicht mehr, und die Politik der EZB kann sich nicht an länderspezifischen Inflationsraten orientieren. Auch deshalb gab es aus Sicht einer offenbar eher kurzfristig orientierten Fiskalpolitik kein Motiv, inflatorischen Tendenzen und ihren Folgen durch einen antizyklischen Kurs entgegenzuwirken.

So lässt sich leicht erklären, warum dieser Prozess aus überdurchschnittlicher Preissteigerung und realem Wachstum nicht nur lange anhalten konnte, sondern es auch immer die gleichen Länder waren, in denen er zu beobachten war. Dieser Prozess ist bei gemeinsamer, stabilitätsorientierter Geldpolitik aber nur deshalb möglich gewesen, weil es in der WWU andere Mitgliedstaaten gab - insbesondere ist hier Deutschland zu nennen -, die entsprechende Gegengewichte schufen. Aufgrund gedämpfter oder sogar negativer Lohnstückkostenentwicklung blieb die Inflation in Deutschland meist deutlich unterhalb des Preisziels der EZB von „unter, aber nahe $2 \%$ “. Dies sorgte dafür, dass im Durchschnitt der WWU das Preisziel der EZB nie 
wesentlich überschritten wurde. Im Ergebnis wirkte dieselbe Geldpolitik für Länder mit überdurchschnittlicher Preissteigerung zu expansiv, für Länder mit unterdurchschnittlicher Preissteigerung zu restriktiv.

Es gibt jedoch eine Größe, die - anders als alle zuvor genannten - nicht jährlich wiederkehrend in etwa gleicher Größenordnung ausfiel, sondern kumulativ von Jahr zu Jahr weiter anstieg: das Differential in der preislichen Wettbewerbsfähigkeit, z. B. zwischen Spanien und Deutschland. Zusammen mit einem permanenten Differential zwischen den Wachstumsraten der Binnennachfrage beider Länder führte der kumulierte Unterschied in der Wettbewerbsfähigkeit dazu, dass in Deutschland der Leistungsbilanzüberschuss und in Spanien das Leistungsbilanzdefizit expandierten, hier zuletzt auf einen zweistelligen Anteil am BIP.

Auch die Entstehung und der politische Umgang mit den Divergenzen im Euroraum sind aus der jeweiligen Sichtweise sowie Ausgangs- und Interessenlage zu beurteilen. Alle Länder wurden jährlich in mehrfacher Weise in den Gremien der EU geprüft, so z.B. im Rahmen der Länderexamina des WPA oder anlässlich der Prüfung des Stabilitätsprogramms durch WFA, Eurogruppe und ECOFIN-Rat. Es gab und gibt nach wie vor auch eine klare "Leitlinie" für die Ausrichtung und Prüfung der Lohnentwicklung, auf die sich im Rahmen der „Grundzüge der Wirtschaftspolitik" die Mitgliedstaaten und die Gemeinschaft verpflichtet haben und die nahezu unverändert seit Jahren dort enthalten ist. ${ }^{4}$ Demnach sollen die Mitgliedstaaten auf Rahmenbedingungen hinwirken, die unter Respektierung der Rolle der Sozialpartner zu Nominallöhnen führen, die mit Preisstabilität und der mittelfristigen Produktivitätsentwicklung vereinbar sind. Gemessen daran hat eine ganze Reihe von Ländern - darunter z. B. Spanien - diese Leitlinie tendenziell überschossen. Andere Länder, darunter Deutschland, blieben hinter der Leitlinie zurück. Man könnte es auch so ausdrücken: Einige Länder haben sich der gemeinsamen Geldpolitik inflatorisch zur Stärkung ihrer Binnendynamik bedient, andere Länder haben ihre Binnennachfrage begrenzt und das Regime der unwiderruflich fixierten Wechselkurse über eine reale Abwertung zur Verbesserung der preislichen Wettbewerbsfähigkeit genutzt. Wurden diese Divergenzen in früheren Jahren durch eine WWU-weite Prosperität zeitweise überspielt, zeigen sie sich in Zei- ten der Krise mit aller Härte als nicht mehr fortsetzbar. Inzwischen werden Länder mit beeinträchtigter Wettbewerbsfähigkeit und hohen Leistungsbilanzdefiziten durch empfindliche Zinsaufschläge auf ihre Staatanleihen im Vergleich zu den deutschen Staatsanleihen sanktioniert. Demgegenüber finden Länder mit anhaltend ausgeprägten Leistungsbilanzüberschüssen nach dem Einbruch der Weltwirtschaft in der bisher schwachen Binnennachfrage keine Kompensation für den schockartigen Rückgang ihres Außenbeitrags. Die langfristigen Effekte dieser Divergenzen wurden wirtschaftspolitisch über lange Zeit missachtet. Im Vertrauen auf marktmäßige Anpassungsprozesse ist eine rechtzeitige adäquate wirtschaftspolitische Reaktion ausgeblieben. ${ }^{5}$

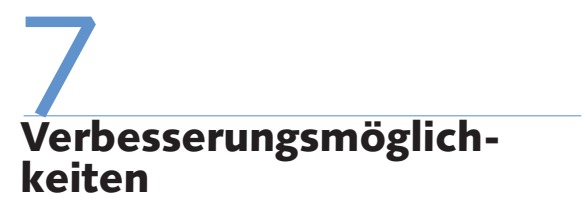

Der MED ist von seiner Zielsetzung und seiner Zusammensetzung her eine Art „Maximum Maximorum“ der makroökonomischen Koordinierung im Euroraum.

Im Lichte der bisherigen Erkenntnisse und Erfahrungen gab es zwischenzeitlich und insbesondere nach zehn Jahren WWU und MED eine Reihe von Vorschlägen zur Verbesserung seiner Wirksamkeit. Zu nennen sind z.B. eine bessere quantitative Fundierung des Gedankenaustauschs, die Einrichtung eines speziellen MED für den Euroraum, eine Verbesserung der Abstimmung zwischen EU- und nationaler Ebene, eine höhere Sichtbarkeit, vor allem aber eine bessere Ausschöpfung seines Koordinierungspotenzials selbst. ${ }^{6}$ In diesem Zusammenhang soll hier insbesondere auf zwei Aspekte eingegangen werden. Der eine zielt auf eine institutionelle, der andere auf eine inhaltliche Stärkung des MED ab.

\subsection{INSTITUTIONELLE STÄRKUNG}

Ein Teil der Vorschläge zur institutionellen Veränderung des Rahmens fordert eine stärkere Einbindung des MED in das Regelwerk der wirtschaftspolitischen Koordination der EU. Ein Anlass war die zwischenzeitliche Debatte um eine Europäische Verfassung. Stellvertretend seien hier die Empfehlungen des Deutschen Gewerkschaftsbundes an den damaligen Europäischen Konvent wiedergegeben (Deutscher
Gewerkschaftsbund 2005). Für den makroökonomischen Policy-Mix und den MED waren dabei vor allem folgende Punkte von Bedeutung:

- Gleichgewichtige Berücksichtigung des Wachstums- und Beschäftigungsziels neben dem Stabilitätsziel in Art. 105 EG-Vertrag, vergleichbar den Bestimmungen des US Federal Reserve Act und des deutschen Stabilitäts- und Wachstumsgesetzes.

- Verankerung des MED im EG-Vertrag durch Ergänzung des Art. 99 Abs. 1 EGVertrag, wonach in die Prüfung der „Grundzüge“ neben dem Beschäftigungsausschuss nach Art. 130 auch der MED einbezogen wird.

- Erweiterung des Zielkatalogs in Art. 99 um das Ziel „spannungsfreier makroökonomischer Policy-Mix im Zusammenwirken von Geld- und Fiskalpolitik sowie der Lohn- und Einkommensentwicklung zur Erlangung eines dauerhaft hohen Beschäftigungsstandes“.

Diese Vorschläge wurden jedoch nicht in den Entwurf der Verfassung aufgenommen. Der Verfassungsprozess selbst scheiterte nach der Ablehnung des Entwurfs in den Referenden in Frankreich und den Niederlanden 2005 und wurde durch das Vertrags-Konzept von Lissabon ersetzt.

\subsection{INHALTLICHE STÄRKUNG}

Vor dem Hintergrund des Status quo bei Institutionen und Vertrag zielen weitere Vorschläge darauf, diesen gegebenen Rah-

4 Leitlinie 4 in der geltenden Fassung der "Grundzüge" lautet: „Member States should encourage the right framework conditions for wage-bargaining systems, while fully respecting the role of the social partners, with a view to promoting nominal wage and labour-cost developments consistent with price stability and the trend in productivity over the medium term, taking into account differences across skills and local labour market conditions." (European Commission 2005, S. 41).

5 Eine Aufforderung zur Korrektur der überschießenden Divergenzen, allerdings nur für Defizitländer, beinhaltet Leitlinie 1 der "Grundzüge" in der geltenden Fassung: „....Member States posting current account deficits that risk being unsustainable should work towards correcting them by implementing structural reforms, boosting external competitiveness and, where appropriate, contributing to their correction via fiscal policies..." (European Commission 2005, S. 41). Zur Thematik der Divergenzen generell existiert eine reichhaltige Literatur, zuletzt z.B. Flassbeck (2009, S. 69) oder European Commission (2009).

6 Siehe Fußnote 2. 
men besser inhaltlich auszufüllen. Durch Verhaltensänderungen der Akteure könnten demnach auch innerhalb der bestehenden Institutionen Fortschritte für Wachstum und Beschäftigung erzielt werden.

Es ist gerade die derzeitige Krise, die hierzu Anstoß geben könnte. Sie zeigt, dass man der Makropolitik - gemessen am Einsatz von Geld- und Fiskalpolitik - zutraut, die konjunkturelle Abwärtsbewegung zu mildern bzw. umzukehren. Diese Einsicht in die Wirkung der Makropolitik gilt es, über die Bewältigung der aktuellen Krise hinaus zu bewahren. Sie sollte dazu führen, die wirtschaftspolitische Agenda neben der Strukturreformpolitik, die die ersten zehn Jahre der WWU, aber auch den Stellenwert des MED zunehmend geprägt hatte, wieder um eine stärkere makroökonomische Dimension zu ergänzen.

Die wirtschaftliche Entwicklung kennt keinen Nullpunkt: Was für die Stabilisierung und die Umkehr der Abwärtsbewegung richtig ist, trägt - wenngleich bei anderer Dosierung der Instrumente - auch weiter über eine nachhaltige Aufwärtsentwicklung bis in die volle Ausschöpfung des vorhandenen Potenzials und seine Stärkung ohne Gefährdung der Preisstabilität. Die Sichtweise darüber, wie eine wachstums-, beschäftigungs- und stabilitätsorientierte gesamtwirtschaftliche Politik angelegt werden kann, sollte nicht nur von Abwehr und Enthaltung, sondern auch von Handeln und Gestaltung geleitet werden. Konkret heißt das:

- Fiskal- und Lohnpolitik als die wesentlichen Akteure, von denen eine "hausgemachte" Verletzung der Preisstabilität herrühren könnte, verpflichten sich, dauerhaft Preisstabilität zu wahren.

- Die Lohnentwicklung in jedem Mitgliedstaat orientiert sich stabilitätsorientiert, verteilungs- und wettbewerbsneutral am länderspezifischen mittelfristigen Produktivitätswachstum und am Preisziel der EZB. Verletzungen dieser Norm in der Vergangenheit sind zu korrigieren und in der Zukunft zu unterlassen.

- Die Geldpolitik sorgt unter diesen Voraussetzungen für die bestmöglichen monetären Bedingungen für mehr Investitionen und Beschäftigung.

Diese Agenda ist ein Beispiel für das zuvor angesprochene, sich ergänzende und verstärkende Zusammenwirken von Makropolitik und Strukturreformen: Die Stabilitätsorientierung der Lohnpolitik senkt die gleichgewichtige oder inflationsstabile
Arbeitslosenquote (NAIRU), die eine Catch all-Größe für die Funktion und Verfassung von Arbeitsmärkten ist. In dem MaBe, wie die NAIRU sinkt, steigt das Produktions- und Beschäftigungspotenzial, das wiederum erst durch möglichst günstige monetäre Bedingungen voll realisiert wird.

Die Umsetzung dieser makroökonomischen Koordinierungsaufgabe als „Zielfunktion unter Einhaltung von Nebenbedingungen" beinhaltet einen Prozess, der bis an die Grenzen des Wachstumspotenzials gehen und diese dabei im Zuge einer starken Investitionsdynamik zugleich weiter herausschieben kann. ${ }^{7}$

Selbst wenn es dabei vorübergehend zu einer Anspannung in der Preisentwicklung als Ausdruck einer temporären Knappheitssituation kommen sollte, weil der „Vorfinanzierung“ der Investitionsdynamik durch möglichst günstige makroökonomische Bedingungen noch nicht unmittelbar ihr voller Kapazitätseffekt gegenübersteht, und selbst wenn die am Markt beobachteten Inflationserwartungen auf derartige Anspannungen hindeuten, muss dieser temporäre Preiseffekt kein Anlass zur Sorge sein. „Soweit damit erhöhte Investitionen Hand in Hand gehen, die die Kapazitäten erweitern, und sich über den anschließenden Angebotsdruck die Preissteigerungen wieder zurückbilden, ist dagegen nichts einzuwenden." (SVR 1984, Ziff. 362). Vor allem aber sorgt die oben eingeforderte stabilitätskonforme Ausrichtung der Lohn- und Fiskalpolitik dafür, dass ein derartiger Einmaleffekt kein Einstieg in eine Preis-Lohn-Spirale wird.

Solange es für die Stabilitätsorientierung von Fiskal- und Lohnpolitik keine institutionell verankerte Zusage gibt, sollte wechselseitiges Vertrauen unter den makroökonomischen Akteuren diese Lücke ausfüllen können. Vertrauen muss oft erst im längeren Miteinander wachsen. Der MED hatte hierfür zehn Jahre Zeit. In diesem Zeitraum kamen informelle, bilaterale Kontakte außerhalb des MED zwischen wichtigen Akteuren wie z.B. der Geld- und Lohnpolitik hinzu. Vor allem aber mit Blick auf das tatsächliche Verhalten der Akteure in dieser Zeit sollte genug Vertrauenskapital entstanden sein, um sich in Zukunft darauf stärker beziehen zu können. Das gilt insbesondere für die faktische Stabilitätsorientierung der Lohnentwicklung, deren Bedeutung von der Geldpolitik immer wieder betont wird und deren Einhaltung von ihr bisweilen auch attestiert wird. ${ }^{8}$ Dabei sollte die Wahrung der Preisstabilität durch die Sozialpartner nicht nur von der Befürchtung getragen sein müssen, dass eine Verletzung durch monetäre Restriktion sanktioniert wird. Mindestens ebenso sollte - im Einklang mit dem EG-Vertrag - die begründete Erwartung bestehen können, dass nachhaltige Stabilitätsorientierung durch eine möglichst starke Unterstützung von Wachstum und Beschäftigung seitens der Geldpolitik honoriert wird. Eine derart offensiv-dynamische Sicht bedeutet nichts anderes als den Auftrag des Europäischen Rates von Köln so umzusetzen, wie er in dessen Entschließung im Jahr 1999 enthalten ist:

„Für ein starkes Beschäftigungswachstum bei Preisstabilität müssen Finanzpolitik, Geldpolitik und Lohnentwicklung dauerhaft spannungsfrei zusammenwirken. Der Europäische Rat fordert alle, die wirtschafts- und beschäftigungspolitische Entscheidungen fällen oder diese beeinflussen, unter voller Anerkennung ihrer Unabhängigkeit und Autonomie in ihrem jeweiligen Verantwortungsbereich auf, zu mehr Beschäftigung auf der Grundlage eines starken und nichtinflationären Wachstums beizutragen. In einem makroökonomischen Dialog auf der Grundlage gegenseitigen Vertrauens sollten in geeigneter Weise Informationen und Meinungen zu der Frage ausgetauscht werden, wie die gesamtwirtschaftliche Politik zu gestalten ist, damit die Möglichkeiten für Wachstum und Beschäftigung ausgebaut und voll ausgeschöpft werden können“ (Europäischer Rat 1999, Ziff. 5).

Es ist zu wünschen, dass in Zukunft bei der Bilanzierung des MED niemand fragen muss, ob das Glas halb leer oder halb voll ist, sondern jeder feststellen kann, dass es, ohne überzuschwappen, bis zum Rand gefüllt ist.

\footnotetext{
7 Ähnliche Formulierungen finden sich in mehreren Fassungen der "Grundzüge “, so bereits 1997: „Je stärker die Geldpolitik bei ihrer Stabilitätsaufgabe durch angemessene Haushaltspolitiken und Lohnentwicklungen entlastet wird, desto eher werden die monetären Rahmenbedingungen, einschließlich der Wechselkurse und der langfristigen Zinsen, Wachstum und Beschäftigung begünstigen " (Rat der Europäischen Union 1997, S. 13).

8 So erklärte der Präsident der EZB auf der Pressekonferenz vom 10. Mai 2001: „.... wage moderation has so far been maintained for the euro area as a whole, despite the effect of the oil price shock. This has been a very positive factor and lends support to those forecasts which assume that current inflation rates will not spill over into future wage negotiations" (Duisenberg 2001).
} 


\section{LITERATUR}

Deutscher Gewerkschaftsbund (German Trade Union Confederation) (2005): A new social contract for Europe - Recommendations to the European Convention, in: Hein, E./Niechoj, T./Schulten, T./Truger, A, (Hrsg.): Macroeconomic Policy Coordination in Europe and the Role of the Trade Unions, Brussels, S. 319-338

Duisenberg, W. F. (2001): EZB-Pressekonferenz vom 10. Mai, Frankfurt/M., http://www.ecb.int/press/pressconf/2008/html/index.en.html Europäischer Rat (1999): Schlussfolgerungen des Europäischen Rates (Köln) vom 3./4. Juni, Anhang I: Entschließung des Europäischen Rates und Bericht über den Europäischen Beschäftigungspakt, http://www. europarl.europa.eu/summits/kol2_de.htm

European Commission (2005): The broad economic policy guidelines (for the 2005-08 period), European Economy N 4/2005, Brussels, http://ec.europa.eu/economy_finance/publications/publication6432_en. pdf

European Commission (2009): Quarterly Report on the Euro Area 1 Directorate General for Economic and Financial Affairs, European Communities, Brussels

Flassbeck, H. (2009): Gescheitert - Warum die Politik vor der Wirtschaft kapituliert, Frankfurt/M

Flassbeck, H./Spiecker, F. (2007): Das Ende der Massenarbeitslosigkeit, Frankfurt/M

Hallwirth, V. (1998): Und Keynes hatte doch recht - Eine neue Politik für Vollbeschäftigung, Frankfurt/New York

Hallwirth, V./Koll, W. (2009): Zehn Jahre Makroökonomischer Dialog eine Zwischenbilanz, in: Wirtschaftsdienst, 1, S. 26-33, http://www. wirtschaftsdienst.eu/archiv/jahr/2009/1/
Holtfrerich, C.-L. (2007): Wo sind die Jobs? - Eine Streitschrift für mehr Arbeit, München

Horn, G. (2005): Die deutsche Krankheit - Sparwut und Sozialabbau, München/Wien

Janssen, R. (2005): Policy coordination in the Macroeconomic Dialogue of Cologne: Experiences from the ETUC, in: Hein, E./Niechoj, T./Schulten, T./Truger, A. (Hrsg.): Macroeconomic Policy Coordination in Europe and the Role of the Trade Unions, Brussels, S. 213-236 Koll, W. (2005): Macroeconomic Dialogue - Development and Intentions, in: Hein, E./Niechoj, T./Schulten, T./Truger, A, (Hrsg.): Macroeconomic Policy Coordination in Europe and the Role of the Trade Unions, Brussels, S. 175-212

Rat der Europäischen Union (1997): Empfehlung des Rates vom 7. Juli 1997 über die Grundzüge der Wirtschaftspolitik der Mitgliedstaaten und der Gemeinschaft, in: Amtsblatt der Europäischen Union L 209, vom 2.8., S. $12-18$

Sachverständigenrat zur Begutachtung der gesamtwirtschaftlichen Entwicklung (SVR) (1984): Chancen für einen langen Aufschwung. Jahresgutachten 1984/85, Wiesbaden

Watt, A. (2005): Can reform of the Macroeconomic Dialogue improve macroeconomic policy-making in Europe?, in: Hein, E./Niechoj, T./Schulten, T./Truger, A, (Hrsg.): Macroeconomic Policy Coordination in Europe and the Role of the Trade Unions, Brussels, S. 237-259 